\title{
A Flexible Wireless Dielectric Sensor for Noninvasive Fluid Monitoring
}

\author{
Heng-Tian Zhu, Ye Chen, Yi-Feng Xiong, Fei Xu * and Yan-Qing Lu \\ College of Engineering and Applied Sciences, Nanjing University, Nanjing 210093, China; \\ mg1834019@smail.nju.edu.cn (H.-T.Z.); yechen@nju.edu.cn (Y.C.); \\ dz1734004@smail.nju.edu.cn (Y.-F.X.); yqlu@nju.edu.cn (Y.-Q.L.) \\ * Correspondence: feixu@nju.edu.cn
}

Received: 18 November 2019; Accepted: 26 December 2019; Published: 27 December 2019

\begin{abstract}
A flexible wireless dielectric sensor is presented here for noninvasively monitoring the permittivity and conductivity of fluids, based on resistor-inductor-capacitor (RLC) resonant circuit and capacitively coupled contactless conductivity detection $\left(C^{4} D\right)$ technique. The RLC sensor consists of one single-turn inductor and one interdigital capacitor. The resonant frequency of the device is sensitive to the surrounding environment, thanks to the electric field leaked out between the interdigital capacitor electrodes. Through the high-frequency structure simulator (HFSS) simulation, and experiments on ethanol/water solutions and $\mathrm{NaCl}$ solutions, it was confirmed that a fluid's permittivity and conductivity could be detected by the return loss curve $\left(\mathrm{S}_{11}\right)$. With great repeatability and stability, the proposed sensor has potential for broad applications, especially in wearable low-cost smart devices.
\end{abstract}

Keywords: dielectric sensor; wireless passive; noninvasive; fluid monitoring

\section{Introduction}

In recent years, resistor-inductor-capacitor (RLC) resonators as a passive wireless sensing device are developing rapidly. RLC resonators have been widely applied for measuring temperature [1], humidity [2], strain [3], and biological analytes [4] because of their high sensitivity and long lifespan. However, the rigid properties of traditional RLC devices limit their usage in sensing fields. Thanks to the development of microfabrication technology and polymer materials, flexible RLC resonators have emerged and show great potential in specific applications such as skin hydration monitoring [5], intraocular pressure monitoring [6], intracranial pressure monitoring [7], alcohol gas detection [8], and bacteria detection [9].

With the rapid development of Internet of Things (IoT) technique, the demands for detecting the dielectric property of fluids noninvasively are growing significantly, such as pharmaceutical composition determination [10], human body fluid composition analysis [11], and food-safety testing [12]. The dielectric property of fluids can be used to reveal the information on its composition and respective concentrations. Capacitively coupled contactless conductivity detection $\left(C^{4} D\right)$ is a recently developed fluid conductivity detection technique. The $\mathrm{C}^{4} \mathrm{D}$ is able to detect analytes such as ions, biomolecules, and proteins [10-17] in fluids, and avoids the electrochemical reaction on electrodes. Thus, it is widely applied in capillary zone electrophoresis (CE) and high performance liquid chromatography (HPLC).

Recently, the $C^{4} \mathrm{D}$ method combined with radiofrequency technique have attracted an increasing interest from researchers. Rogers et al. introduced a stretchable inductor-capacitor(LC)-based sensor for epidermal measurement of the volume of sweat [18]. Ma et al. introduced an LC microfluidic sensor based on Low Temperature Co-Fired Ceramic (LTCC) technology for detecting glucose with 
different concentrations [19]. To achieve results that are more accurate, conductivity of the fluid can be considered, which will also introduce more applications.

Here, we combine RLC resonant circuit with $C^{4} \mathrm{D}$ technique to describe a flexible wireless dielectric sensor for noninvasively monitoring the permittivity and conductivity of fluids. The presented device consists of one single-turn inductor and one interdigital capacitor. The measured data can be easily collected by a vector network analyzer (VNA) with a readout coil through inductive coupling. The resonant frequency amplitude is used to indicate the conductivity change of the fluid to be tested. As the effect of parasitic capacitances is overcome at the resonant frequency, a higher sensitivity of conductivity is obtained [20]. In addition, the fluid's permittivity can be detected by the drift of the resonant frequency. Thus, we realize a flexible wireless dielectric sensor that can measure the conductivity and permittivity of fluids. According to the same principle, the sensor can also detect gas. In the present study, the high-frequency structure simulator (HFSS) results, and the results from experiments with ethanol/water solutions and $\mathrm{NaCl}$ solutions confirm the noninvasive sensing ability of the flexible wireless dielectric sensor. With the advantages of flexibility, wireless, and noninvasive detection capability, the proposed sensor shows great potential for broad applications, such as wearable blood monitoring and real-time water quality detection.

\section{Materials and Methods}

\subsection{Design and Operating Principle}

The principle of the RLC wireless passive sensor is shown in Figure 1a. The energy and signal can be transported because the dielectric sensor and the external reading circuitry are inductively coupled. The sensor circuit, which consist of stable $L_{s}$, variable $C_{S}$, and variable $R_{S}$, is characterized by its resonant frequency $\left(f_{s}\right)$ and quality factor $\left(Q_{s}\right)$ :

$$
\begin{aligned}
& f_{s}=\frac{1}{2 \pi \sqrt{L_{s} C_{s}}}, \\
& Q_{s}=\frac{1}{R_{s}} \sqrt{\frac{L_{s}}{C_{s}}} .
\end{aligned}
$$

A vector network analyzer (VNA) is used to detect the sensor's resonant frequency through the return loss curve $\left(S_{11}\right)$, which is based on Equation (2):

$$
\begin{gathered}
\frac{Z_{\mathrm{S}}-R_{r}}{Z_{\mathrm{S}}+R_{r}} \\
Z_{S}=R_{S}+j 2 \pi f L_{r}\left(1+\frac{k^{2}\left(\frac{f_{s}}{f}\right)^{2}}{1+\frac{j f}{f_{\mathrm{S}} Q_{s}}-\left(\frac{f_{\mathrm{S}}}{f}\right)^{2}}\right),
\end{gathered}
$$

where $k$ is the coupling coefficient of the readout coil and sensor's coil. When $Q_{s} \gg 1$, the $S_{11}$ is minimal near the sensor resonant frequency because power is maximally absorbed by the resonant circuit.

The structure of the proposed dielectric sensor is shown in Figure 1b. The sensor consists of a single-turn inductor and an interdigital capacitor, which are encapsulated above and below by thin layers of polyimide (PI). The whole structure is printed to a polydimethylsiloxane (PDMS) substrate $(30 \mu \mathrm{m})$ so that the sensor can work on an uneven surface. The planar spiral inductor has a $7.5 \mathrm{~mm}$ inner diameter and an $8.6 \mathrm{~mm}$ outer diameter, and the width of the coil is $300 \mu \mathrm{m}$ considering the skin effect. The interdigital electrodes are approximately $700 \mu \mathrm{m}$ long (580 $\mu \mathrm{m}$ in overlapping length) and $180 \mu \mathrm{m}$ wide, with a uniform spacing of $30 \mu \mathrm{m}$ between adjacent electrodes. The digits' number of the interdigital electrodes is adjustable so that the fluid's dielectric property in different frequencies can be discovered.

As shown in Figure 1c, the interdigital capacitor is sensitive to the dielectric property of the surrounding medium because the electric field leaks out between the interdigital electrodes. Thus, the sensor can detect the permittivity and conductivity of the fluid noninvasively, as shown in Figure 1d. 
In the equivalent circuit in Figure $1 \mathrm{~d}$, the $C_{p}$ represents the capacitance when the electrical field passes through the air and PDMS membrane. The $C_{1}$ and $C_{2}$ represent the capacitance when the electrical field passes through the tube. The fluid can be considered as a resistance parallel connection with a capacitor due to its impedance and capacitive reactance, which cannot be ignored at radio frequency. The change of the fluid's permittivity affects the integral polarization of the surrounding medium, thus influences the capacitance of the sensor. This leads to a drift of the resonant frequency. In addition, the conductivity of the fluid interferes with the electromagnetic coupling between the sensor and the readout coil, resulting in a variation of the peak amplitude of $S_{11}$.

(a)

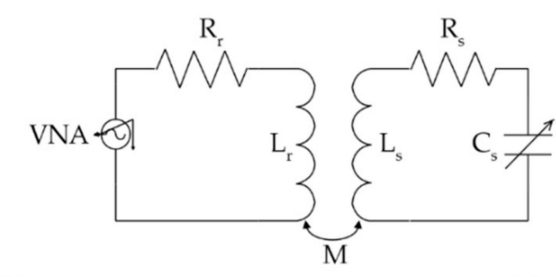

(c)

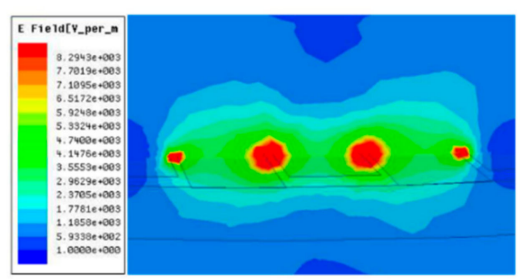

(b)

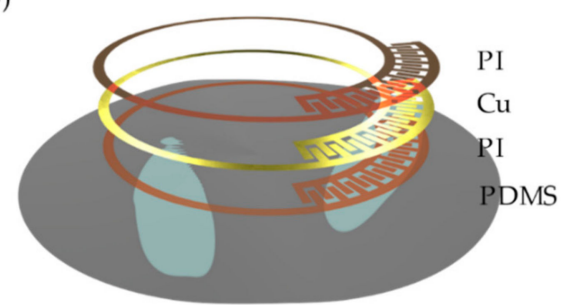

(d)

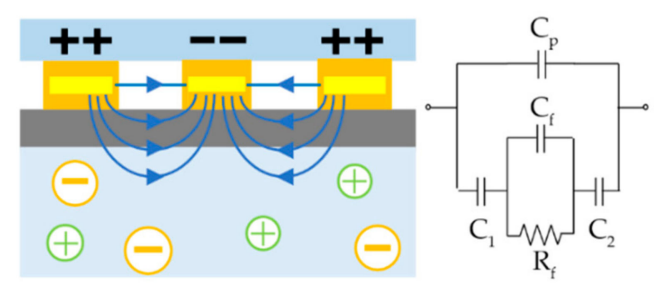

Figure 1. (a) Illustrative model schematic of resistor-inductor-capacitor (RLC) wireless measurement; (b) structural schematic of the proposed dielectric sensor; (c) electric field leaked out between the interdigital electrodes on high-frequency structure simulator (HFSS) simulation; and (d) schematic and equivalent circuit of the fluid monitoring.

\subsection{Sensor Fabrication}

In this work, we fabricated the proposed dielectric sensor using transfer printing technology, [21] which achieves a highly compatible assembly of micro/nanostructured materials on elastomers and has the advantages of excellent repeatability, simple operation, and low cost. Figure 2 illustrates the general process of fabrication.

The fabrication starts with spin coating a layer of polyimide $(1 \mu \mathrm{m})$ onto glass at $3000 \mathrm{rpm}$. After amination at $250{ }^{\circ} \mathrm{C}$ for $40 \mathrm{~min}$, a layer of photoresist (PR) is spin-coated on the PI layer and exposed to define the pattern of the inductor and interdigital capacitor. A Cu layer $(2 \mu \mathrm{m})$ is electron-beam deposited on the PI layer using the standard lift-off technique. Then, another layer of polyimide $(1 \mu \mathrm{m})$ is spin-coated at $3000 \mathrm{rpm}$ and aminated at $120^{\circ} \mathrm{C}$ for $30 \mathrm{~min}$ to serve as the top layer. After that, the bottom and top PI layers can be patterned using the photolithography process and etched by oxygen plasma to obtain a micro-sized pattern so that the sensor is on the glass slide. Before transfer printing, the elastomeric substrate (PDMS) is preprocessed by oxygen plasma for $2 \mathrm{~min}$ to clean and activate its surface. Then, the sensor can be picked up directly by the thermal release tape (TRT) and printed to the PDMS substrate by a heating step [21]. 


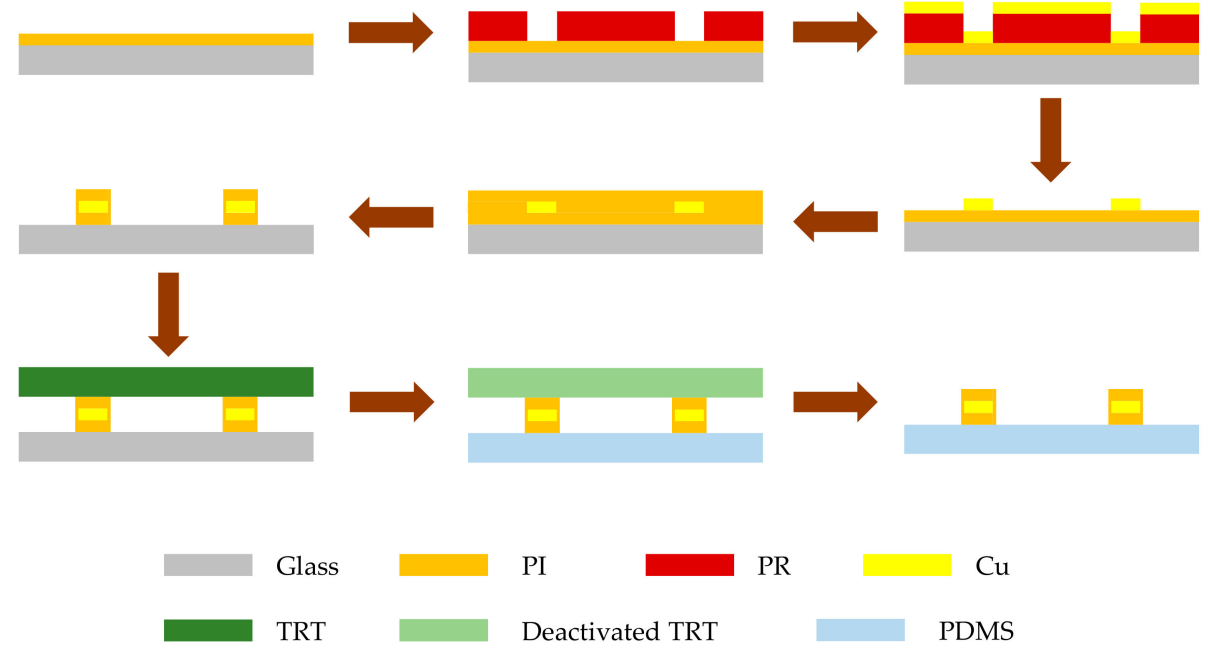

Figure 2. Fabrication process of the proposed RLC dielectric sensor.

\section{Results}

\subsection{Simulation of the Dielectric Sensor}

As shown in Figure 3a, we simulated the dielectric sensor noninvasively monitoring a fluid in HFSS. The readout coil was set $3 \mathrm{~mm}$ above the sensor to transfer energy and detect the signal. The cylindric fluid was installed $0.3 \mathrm{~mm}$ below the sensor with insulating medium between them. The digits' number of the interdigital electrodes was adjusted from 12 to 24 and the sensor's resonant frequency was changed from 3.47 GHz to $2.77 \mathrm{GHz}$ (Figure 3b). The trend of the resonant frequency drift was different when the number of digits was 20 due to the asymmetry of the structure, where the distance between the interdigital electrodes and the fluid increased a lot when the number of digits was 20. It can be concluded that by adjusting the sensor digits' number, a fluid's dielectric property can be detected at different frequencies so that more information is obtained for big data analytics.

More importantly, we considered the influence of the permittivity and the conductivity of the fluid respectively. We used a 24-digit sensor to detect the fluid of different relative permittivities and conductivities. As shown in Figure $3 c, d$, the conductivity of the fluid was set to zero and the relative permittivity was adjusted from 70 to 10 . The decreased relative permittivity reduced the interdigital electrodes' capacitance, and then increased the sensor's resonant frequency from 2.733 to $2.752 \mathrm{GHz}$. Figure 3e,f shows that the relative permittivity of the fluid was set to 80 and the conductivity was adjusted from 0 to $10 \mathrm{~S} / \mathrm{m}$. The increased conductivity improved the medium's polarization, so the interdigital electrodes' capacitance was enhanced. The increased conductivity weakens the electromagnetic coupling between the readout coil and the sensor, decreasing the sensor's resonant frequency [5] from 2.757 to $2.733 \mathrm{GHz}$ and the amplitude of the $\mathrm{S}_{11}$ peak from 4.21 to $2.01 \mathrm{~dB}$. 
(a)

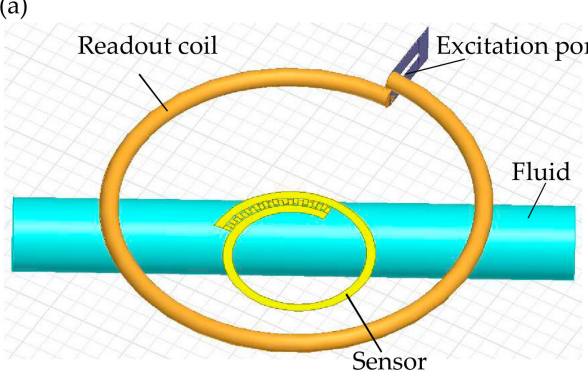

(c)

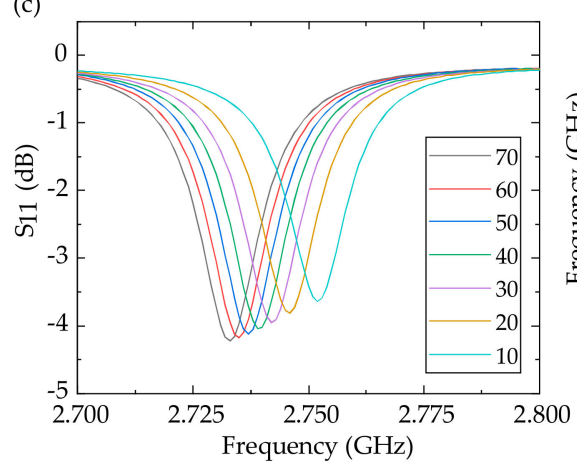

(e)

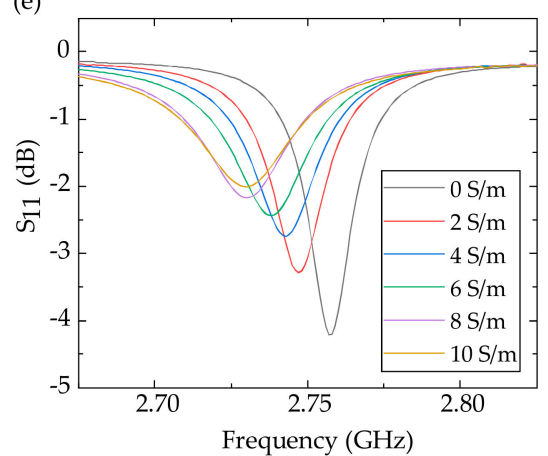

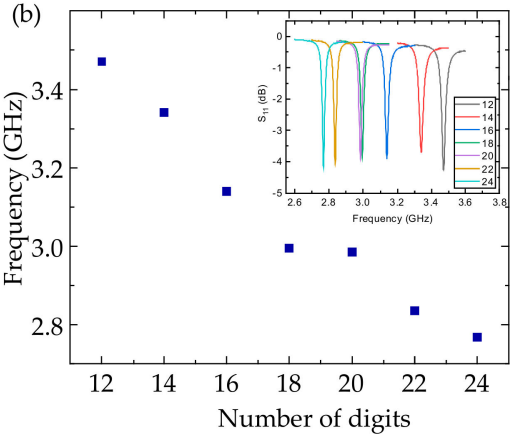
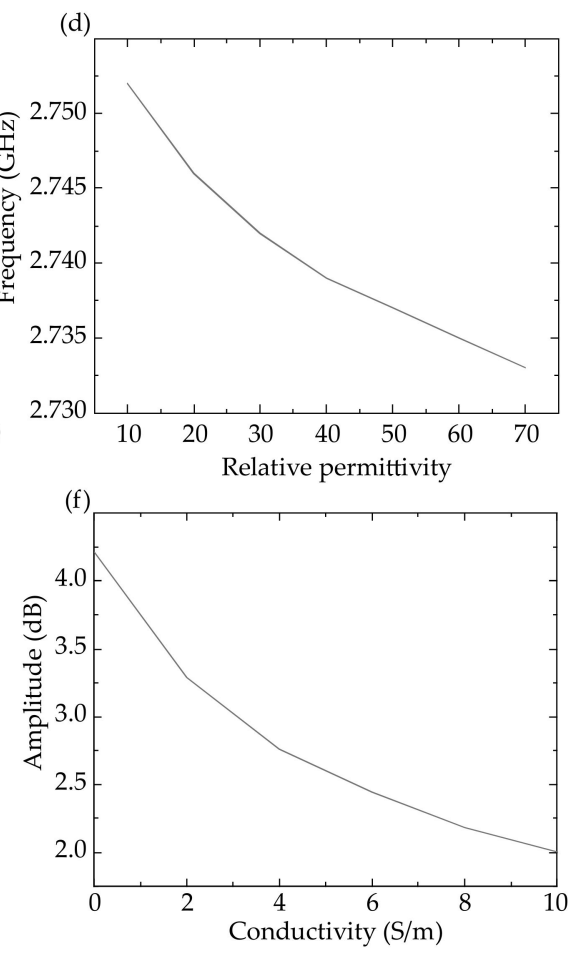

Figure 3. HFSS simulation of the dielectric sensor monitoring the fluid. (a) Model of the HFSS simulation and (b) the sensor's resonant frequency with 12-24 digits. The $S_{11}$ (c) and fitting results (d) of the fluid with different relative permittivities. The $S_{11}$ (e) and fitting results (f) of the fluid with different conductivities.

\subsection{Monitoring the Dielectric Property of the Ethanol/Water Solutions}

In the experiment, we fabricated a 24-digit dielectric sensor and pasted the interdigital electrode on the outside wall of a silicone tube ( $6 \mathrm{~mm}$ outer diameter and $1 \mathrm{~mm}$ thickness). The readout coil was $3 \mathrm{~mm}$ above the sensor and connected to the VNA via an external coaxial cable, as is shown in Figure $4 \mathrm{a}, \mathrm{b}$. The fluid with different dielectric property was injected into the tube using a peristaltic pump. Between adjacent tests, the previous tested liquid was pumped out and the tube was washed twice by pure water. After pumping out the pure water, the tube was blown dry to ensure the sensor's resonant frequency recovered to the initial value. Note that all the tests were conducted at room temperature. 
(a)

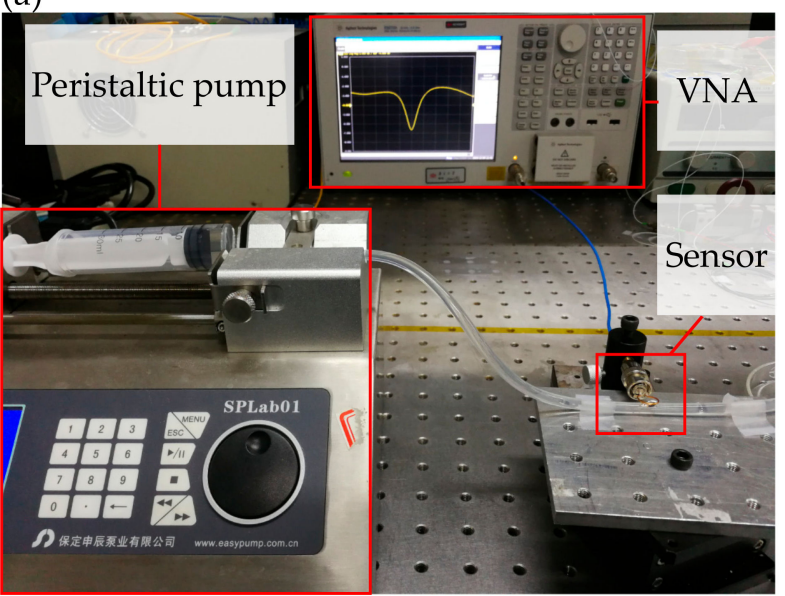

(b)

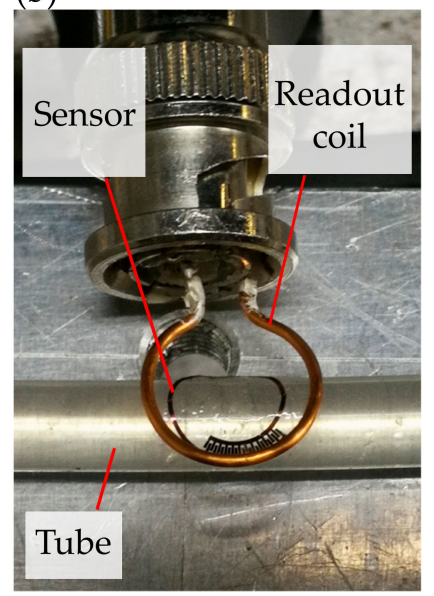

Figure 4. Experimental setup of the dielectric sensor monitoring the fluid. (a) Picture of the testing platform for the dielectric sensor. (b) Picture of the sensor stuck on the tube and communicated with the readout coil.

First, ethanol/water solutions with ethanol's volume fraction of $0 \%, 20 \%, 40 \%, 60 \%, 80 \%$, and $100 \%$ were injected into the tube. The relative permittivity of the ethanol/water solution was changed from 68 to 11 [22] and the conductivity was changed from 0.90 to $1.08 \mathrm{~S} / \mathrm{m}$ [23] with the ethanol's volume fraction adjusted from 0 to 1 at the 2-GHz frequency. Because of the decrease in the permittivity, the sensor's capacitance was reduced, resulting in an increase in the resonant frequency from 1.855 to $1.861 \mathrm{GHz}$. Therefore, we can detect the ethanol's volume fraction in ethanol/water solution with a sensitivity of about $6.56 \mathrm{MHz}$ per unit volume fraction. The average standard deviation was $0.193 \mathrm{MHz}$ (about $1.04 \%$ in relative) among four measurements, which indicated excellent repeatability of the dielectric sensor (in Figure $5 a, b$ ). The response curve was not linear due to the existence of $C_{p}, C_{1}$, and $C_{2}$ (in Figure 1d). Furthermore, we considered the sensor's stability when pure water was injected (in Figure 5c). The frequency drifted between 1.85425 and $1.85563 \mathrm{GHz}$ (about 3.72\%o in relative) within 2 $\mathrm{h}$, showing great stability. The stability will be improved once the sensor is appropriately protected against the effect of environmental humidity.
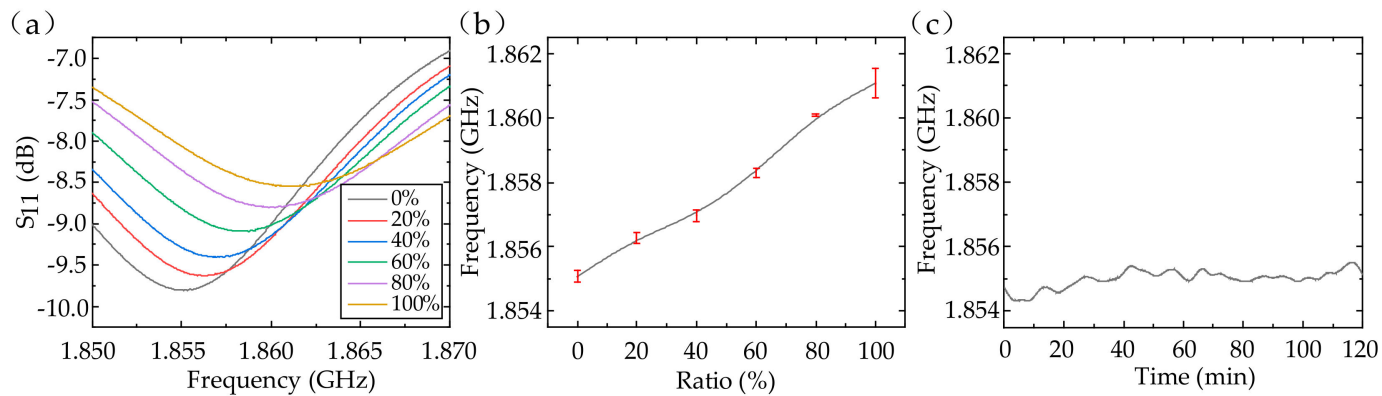

Figure 5. The $S_{11}$ (a) and fitting results (b) of the ethanol/water solutions with different ethanol's volume fraction. (c) Sensor's stability with pure water injected.

\subsection{Monitoring the Dielectric Property of $\mathrm{NaCl}$ Solutions}

The most prominent application for conductivity characterization of fluids is the ion-concentration measurement. We detected the $\mathrm{NaCl}$ solution's dielectric property using a 24-digit sensor. $\mathrm{NaCl}$ solutions with different concentrations from 0 to $1 \mathrm{M}$ were injected into the tube. The relative permittivity of the $\mathrm{NaCl}$ solution changed from 79 to 67 and the conductivity increased from 0 to $7.8 \mathrm{~S} / \mathrm{m}$ with the concentration adjusted from 0 to $1 \mathrm{M}$ at the 2-GHz frequency [24]. Because of the decrease in the electromagnetic coupling, the amplitude of the $S_{11}$ peak declined from 10.092 
to $8.582 \mathrm{~dB}$. Therefore, we can detect the concentration of the $\mathrm{NaCl}$ solution with a sensitivity of about $-1.38 \mathrm{~dB} / \mathrm{M}$. The average standard deviation was $0.00531 \mathrm{~dB}$ (about $5.69 \%$ in relative) among four measurements, which indicated excellent repeatability of the dielectric sensor (in Figure 6a,b). The resonant frequency was relatively fixed, because of the neutralization between the increase of the resonant frequency caused by the solution's permittivity and the decrease of the resonant frequency caused by the electromagnetic coupling. Furthermore, we considered the sensor's stability when $0.5 \mathrm{M}$ $\mathrm{NaCl}$ solution was injected (in Figure 6c). The amplitude drifted between 8.979 and $9.025 \mathrm{~dB}$ (about $25.55 \%$ o in relative) within $2 \mathrm{~h}$, showing great stability.
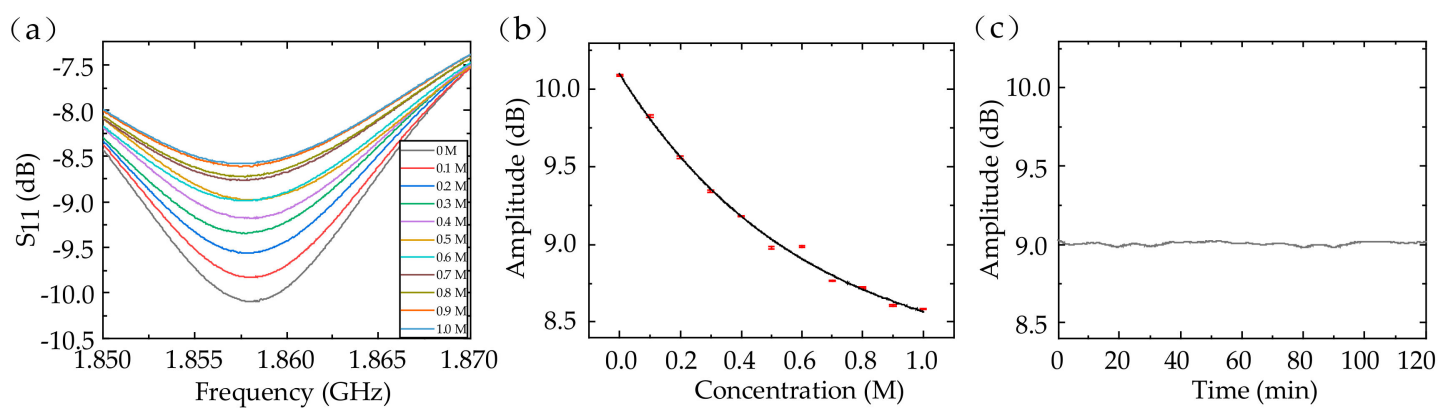

Figure 6. The $\mathrm{S}_{11}$ (a) and fitting results (b) of the $\mathrm{NaCl}$ solutions with different concentrations. (c) The sensor's stability with $0.5 \mathrm{M} \mathrm{NaCl}$ solution injected.

HFSS software simulation and the experiments demonstrated that the fluid's permittivity and conductivity mainly influenced the sensor's capacitance and electromagnetic coupling, respectively, and then changed the $S_{11}$. Therefore, we can noninvasively detect the concentration of a known species that affects the dielectric property of the fluid.

\section{Conclusions}

A novel flexible wireless passive dielectric sensor for noninvasive fluid monitoring was proposed in this paper. The sensor is based on RLC resonant circuitry, consisting of one single-turn inductor and one interdigital capacitor with an adjustable digits' number. Therefore, the sensor's resonant frequency can be regulated and more information can be obtained for big data analytics. The sensor is fabricated on glass and transferred to a PDMS substrate by thermal release tape so that it is flexible and can work on an uneven surface, such as human skin or a pipe. Through HFSS simulation and experiment, it is determined that a fluid's permittivity influences the sensor's capacitance, expressed as the drifted resonant frequency. Furthermore, fluid's conductivity mostly influences the electromagnetic coupling, expressed as the drifted peak amplitude. Thus, we can noninvasively detect the concentration of a known species which affects the dielectric property of the fluid, such as the ethanol/water solution (with a sensitivity of $6.56 \mathrm{MHz}$ ), and $\mathrm{NaCl}$ solution (with a sensitivity of $-1.38 \mathrm{~dB} / \mathrm{M}$ ). The sensitivity will increase with thinner tube's wall. Moreover, it is feasible that the fluid's permittivity and conductivity can be demodulated simultaneously from the return loss curve's resonant frequency and peak amplitude. Having great repeatability and stability, the sensor shows great potential for broad applications, such as real-time blood monitoring and real-time water quality detection.

Author Contributions: H.-T.Z. fabricated the sensors and performed the measurements. H.-T.Z. and Y.C. analyzed the data and wrote the main manuscript text. The experiments of the sensor were completed with the help from Y.C., Y.-F.X., F.X. and Y.-Q.L. All authors reviewed the manuscript. All authors contributed to discussions and writing of the manuscript. F.X. supervised the research. All authors have read and agreed to the published version of the manuscript.

Funding: This research was funded by National Natural Science Foundation of China (No. 61535005 and 61475069), National Science and Technology Major Projects (No. 2017 YFC1403803 and 2017YFA0303700), Shenzhen Science and Technology Research Funding (No. JCYJ20180307155025481), the Fundamental Research Funds for the Central Universities (No. 14380160).

Conflicts of Interest: The authors declare no conflict of interest. 


\section{References}

1. Dan, Y.Y.; Yang, Y.Y.; Hong, Y.; Liang, T.; Yao, Z.; Chen, X.; Xiong, J. Low-Cost Wireless Temperature Measurement: Design, Manufacture, and Testing of a PCB-Based Wireless Passive Temperature Sensor. Sensors 2018, 18, 532.

2. Su, S.; Lv, W.; Zhang, T.; Tan, Q.; Zhang, W.; Xiong, J. A MoS2 Nanoflakes-Based LC Wireless Passive Humidity Sensor. Sensors 2018, 18, 4466. [CrossRef] [PubMed]

3. Butler, J.C.; Vigliotti, A.J.; Verdi, F.W.; Walsh, S.M. Wireless, passive, resonant-circuit, inductively coupled, inductive strain sensor. Sens. Actuators A (Phys.) 2002, 102, 61-66. [CrossRef]

4. Kim, N.-Y.; Adhikari, K.; Dhakal, R.; Chuluunbaatar, Z.; Wang, C.; Kim, E.-S. Rapid, Sensitive, and Reusable Detection of Glucose by a Robust Radiofrequency Integrated Passive Device Biosensor Chip. Sci. Rep. 2015, 5, 7807. [CrossRef]

5. Huang, X.; Liu, Y.; Cheng, H.; Shin, W.-J.; Fan, J.A.; Liu, Z.; Lu, C.J.; Kong, G.-W.; Chen, K.; Patnaik, D.; et al. Materials and Designs for Wireless Epidermal Sensors of Hydration and Strain. Adv. Funct. Mater. 2014, 24, 3846-3854. [CrossRef]

6. Kim, J.; Kim, M.; Lee, M.-S.; Kim, K.; Ji, S.; Kim, Y.-T.; Park, J.; Na, K.; Bae, K.-H.; Kim, H.K.; et al. Wearable smart sensor systems integrated on soft contact lenses for wireless ocular diagnostics. Nat. Commun. 2017, 8, 14997. [CrossRef]

7. Chen, L.Y.; Tee, B.C.; Chortos, A.L.; Schwartz, G.; Tse, V.; Lipomi, D.J.; Wong, H.S.; McConnell, M.V.; Bao, Z. Continuous wireless pressure monitoring and mapping with ultra-small passive sensors for health monitoring and critical care. Nat. Commun. 2014, 5, 5028. [CrossRef]

8. Kim, S.-Y.; Kim, J.; Cheong, W.H.; Lee, I.J.; Lee, J.; Im, H.-G.; Kong, H.; Bae, B.-S.; Park, J.-U. Alcohol gas sensors capable of wireless detection using In2O3/Pt nanoparticles and Ag nanowires. Sens. Actuators B Chem. 2018, 259, 825-832. [CrossRef]

9. Mannoor, M.S.; Tao, H.; Clayton, J.D.; Sengupta, A.; Kaplan, D.L.; Naik, R.R.; Verma, N.; Omenetto, F.G.; McAlpine, M.C. Graphene-based wireless bacteria detection on tooth enamel. Nat. Commun. 2012, 3, 763. [CrossRef]

10. Kubáň, P.; Hauser, P.C. Contactless conductivity detection for analytical techniques-Developments from 2012 to 2014. ELECTROPHORESIS 2015, 36, 195-211. [CrossRef]

11. Kubáň, P.; Hauser, P.C. Contactless conductivity detection for analytical techniques-Developments from 2014 to 2016. ELECTROPHORESIS 2017, 38, 95-114. [CrossRef]

12. Kubáň, P.; Hauser, P.C. Contactless conductivity detection for analytical techniques: Developments from 2010 to 2012. ELECTROPHORESIS 2013, 34, 55-69. [CrossRef]

13. Fracassi, S.; José, A.; do Lago, C.L. An Oscillometric Detector for Capillary Electrophoresis. Anal. Chem. 1998, 70, 4339-4343. [CrossRef]

14. Zemann, A.J.; Schnell, E.; Volgger, D.; Bonn, G.K. Contactless Conductivity Detection for Capillary Electrophoresis. Anal. Chem. 1998, 70, 563-567. [CrossRef]

15. Kubáň, P.; Hauser, P.C. A review of the recent achievements in capacitively coupled contactless conductivity detection. Anal. Chim. Acta 2008, 607, 15-29. [CrossRef]

16. Pumera, M. Contactless Conductivity Detection for Microfluidics: Designs and Applications. Talanta 2008, 74, 358-364. [CrossRef]

17. Coltro, W.K.T.; Rodrigo, S.N.; de Jesus, M.A.; Da Silva, J.A.F.; Carrilho, E. Microfluidic devices with integrated dual-capacitively coupled contactless conductivity detection to monitor binding events in real time. Sens. Actuators B Chem. 2014, 192, 239-246. [CrossRef]

18. Huang, X.; Liu, Y.; Chen, K.; Shin, W.-J.; Lu, C.-J.; Kong, G.-W.; Patnaik, D.; Lee, S.-H.; Cortes, J.F.; Rogers, J.A. Stretchable, wireless sensors and functional substrates for epidermal characterization of sweat. Small 2014, 10, 3083-3090. [CrossRef]

19. Liang, Y.; Ma, M.; Zhang, F.; Liu, F.; Liu, Z.; Wang, D.; Li, Y.; An, L.C. Wireless Microfluidic Sensor Based on Low Temperature Co-Fired Ceramic (LTCC) Technology. Sensors 2019, 19, 1189. [CrossRef]

20. Shih, C.Y.; Li, W.; Zheng, S.Y.; Tai, Y.C. A Resonance-Induced Sensitivity Enhancement Method for Conductivity Sensors. In Proceedings of the 5th IEEE Conference on Sensors, Daegu, Korea, 22-25 October 2006. 
21. Yan, Z.; Pan, T.; Xue, M.; Chen, C.; Cui, Y.; Yao, G.; Huang, L.; Liao, F.; Jing, W.; Zhang, H.; et al. Thermal Release Transfer Printing for Stretchable Conformal Bioelectronics. Adv. Sci. (Weinh.) 2017, 4, 1700251. [CrossRef]

22. Mashimo, S.; Umehara, T.; Redlin, H. Structures of water and primary alcohol studied by microwave dielectric analyses. J. Chem. Phys. 1991, 95, 6257-6260. [CrossRef]

23. Artemkina, M.Y.; Shcherbakov, V.V.; Korotkova, E.N. High-frequency conductivity of mixtures of water with methanol, ethanol, and propanol. Russ. J. Electrochem. 2015, 51, 180-184. [CrossRef]

24. Peyman, A.; Gabriel, C.; Grant, E.H. Complex permittivity of sodium chloride solutions at microwave frequencies. Bioelectromagnetics 2007, 28, 264-274. [CrossRef] [PubMed]

(C) 2019 by the authors. Licensee MDPI, Basel, Switzerland. This article is an open access article distributed under the terms and conditions of the Creative Commons Attribution (CC BY) license (http://creativecommons.org/licenses/by/4.0/). 